OPEN ACCESS

Edited by:

Kun Sun,

Shenzhen Bay Laboratory, China

Reviewed by:

Nana Jin,

Codex Genetics Limited, China

Antonio Russo,

University of Palermo, Italy

*Correspondence: Xin Hong

hongx@sustech.edu.cn

Specialty section:

This article was submitted to

$R N A$,

a section of the journa

Frontiers in Genetics

Received: 01 July 2021 Accepted: 02 August 2021 Published: 25 August 2021

Citation:

Liu J, Lian J, Chen Y, Zhao X,

Du CZ, Xu Y, Hu H, Rao H and Hong X (2021) Circulating Tumor Cells

(CTCs): A Unique Model of Cancer

Metastases and Non-invasive

Biomarkers of Therapeutic Response.

Front. Genet. 12:734595

doi: 10.3389/fgene.2021.734595

\section{Circulating Tumor Cells (CTCs): A Unique Model of Cancer Metastases and Non-invasive Biomarkers of Therapeutic Response}

\author{
Jialing Liu', Jingru Lian', Yafei Chen', Xin Zhao', ChangZheng Du', Yang Xu', \\ Hailiang Hu${ }^{1}$, Hai Rao ${ }^{1}$ and Xin Hong ${ }^{1 *}$ \\ 1 Department of Biochemistry, School of Medicine, Southern University of Science and Technology, Shenzhen, China, \\ ${ }^{2}$ Department of Hepatobiliary Surgery, The Second Affiliated Hospital and School of Medicine, Southern University \\ of Science and Technology, Shenzhen, China
}

Late-stage cancer metastasis remains incurable in the clinic and is the major cause death in patients. Circulating tumor cells (CTCs) are thought to be metastatic precursors shed from the primary tumor or metastatic deposits and circulate in the blood. The molecular network regulating CTC survival, extravasation, and colonization in distant metastatic sites is poorly defined, largely due to challenges in isolating rare CTCs. Recent advances in CTC isolation and ex vivo culture techniques facilitates single-cell omics and the development of related animal models to study CTC-mediated metastatic progression. With these powerful tools, CTCs can potentially be used as non-invasive biomarkers predicting therapeutic response. These studies may open a new avenue for CTC-specific drug discoveries. In this short review, we aim to summarize recent progress in the characterization of CTCs and their clinical relevance in various cancers, setting the stage for realizing personalized therapies against metastases.

Keywords: circulating tumor cells, cancer metastasis, non-invasive biomarker, therapeutic response, liquid biopsy, microfluidic engineering

\section{INTRODUCTION}

Cancer remains the leading cause of death worldwide, with the number rolling up to approximately 10 million in 2021, which is expected to be further doubled by the year 2040 (Sung et al., 2021). These striking numbers suggest a pressing need to obtain a comprehensive understanding of cancer etiology and the underlying molecular mechanisms that drive metastasis. The detection and treatment of malignant cancers remain largely ineffective due to inter-patient and intra-tumor heterogeneity, as well as the abilities of cancer cells to disseminate locally or distantly through metastatic progression, resulting in treatment failure and high mortality (Nguyen et al., 2009; Hanahan and Weinberg, 2011). Therefore, there is dire need for disruptive medical technologies that can achieve more reliable cancer diagnosis, higher therapeutic efficacies, and more favorable long-term clinical benefit.

Cancer metastasis is considered a multi-step process involving remodeling of distinct key signaling pathways at each stage (Nguyen et al., 2009; Lambert et al., 2017). They include (1) local invasion of primary tumor cells into neighboring sites; (2) intravasation: migration into nearby blood vessels named "intravasation"; (3) transient traveling and survival in the blood circulation, 
a population known as "circulating tumor cells (CTCs)"; (4) extravasation: CTCs travel out of the blood vessel and seeding in distant organs, a process named "extravasation"; (5) colonization and growth in the distant sites, that eventually become clinically detectable metastatic tumors. During this process, many metastatic lesions may be present as micrometastases for a long time, which is difficult to detect clinically, yet they appear to be responsible for the eventual disease relapse (Pantel et al., 2009). The therapeutic development that can selectively and effectively target metastatic disease has been limited since tumors are constantly evolving with dynamic genetic and epigenetic alterations during the transitions from a primary tumor to metastases. Although primary tumor biopsies are relatively accessible, they offer very limited information on the behaviors of metastatic tumors, which are often difficult to obtain by surgery. Unfortunately, most of our current treatment strategies are based on the molecular and pathophysiological information derived from primary tumors that may have limited efficacies on metastatic populations due to significant differences between these two groups. Furthermore, remarkable metastatic heterogeneity exists when tumors shed into circulation followed by colonization to distant organs with distinct microenvironment, which makes it extremely challenging to devise a common treatment strategy to simultaneously eliminate different metastatic populations (McGranahan and Swanton, 2017; Hunter et al., 2018). In this context, CTCs, a unique group of cancer cells generated by tumors shedding from primary or metastatic deposits and enter blood circulation, are potential metastatic precursors that give rise to distinct metastatic populations. CTCs could be non-invasively accessible through simple blood draws and thus may offer as a real-time "liquid biopsy" for probing the biological evolutions of cancer during its transition towards metastases (Haber and Velculescu, 2014; Pantel and Speicher, 2016; Alix-Panabieres, 2020).

CTCs are extremely rare, about one in a billion blood cells may be a CTC. This has imposed a significant technical challenge to the efficiently isolate CTCs. The detection and enrichment technologies of CTCs can be broadly categorized into two types (Yu et al., 2011; Ferreira et al., 2016). The first one is positive selection, mostly relying on antibodies capturing the surface tumor antigen expressed on CTCs. One representative example is the CellSearch System utilizing Epithelial Cell Adhesion Molecule (EpCAM)-based CTC capture and enumeration (Riethdorf et al., 2007). Other positive selection-based CTC enrichment technologies involving antibody or peptidefunctionalized nanoparticle-based CTC capture followed by fluorescent imaging and enumeration (Li et al., 2019). Although antigen-based CTC detection approaches are relatively sensitive, they may miss a significant fraction of CTC population with the change in expression of some antigens (like EpCAM) during epithelial-to-mesenchymal transitions in breast CTCs (Yu et al., 2013; Papadaki et al., 2019). Thus, antigen-independent negative selection methods appear to offer better coverage of these CTC populations. The negative selection methods are designed to achieve efficient depletion of blood components such as red blood cells, platelets, and white blood cells, using sizebased filtration methods or immuno-magnetic depletion using antibodies against common leukocyte markers (Kallergi et al., 2016; Banko et al., 2019). The emergence of several microfluidicbased isolation platforms that can integrate multiple physical and biochemical properties of CTCs into one device allows efficient isolation of CTCs in several cancer types (Jackson et al., 2017; Lin et al., 2020; Pei et al., 2020). One representative example is the CTC-iChip microfluidic system invented by researchers from the Massachusetts General Hospital (Ozkumur et al., 2013; Karabacak et al., 2014). Due to the highly efficient depletion of antibody-tagged white blood cells and size-based sorting strategy, individual viable CTCs and CTC clusters from multiple cancer types could be recovered by the CTC-iChip system in an antigen-independent manner. Isolated CTCs could be subjected for ex vivo culture or in vivo passage in immunocompromised mice, forming CTC cell lines and CTC-derived animal explant models (CDX), respectively, which serve as powerful tools for mechanistic investigation of CTC biology (Yu et al., 2014; Drapkin et al., 2018; Hong et al., 2021).

CTCs are thought to be pre-metastatic populations that give rise to distant metastases, thus a complete understanding of CTC biology may hold the promise for devising new therapeutic strategies for treating metastatic disease. On the contrary, primary tumor biopsy analyses may only reveal signatures that are confined to local tumors, which may have undergone dramatic molecular remodeling during metastatic progression. Second, tissue biopsies are generally limited by surgical accessibilities while CTC collection can be achieved through a simple blood draw in patients. Since such analyses may be repeated before and after therapies in a longitudinal manner to monitor disease progression and therapeutic response, CTC-based liquid biopsy technologies could potentially enable a highly precise and personalized cancer treatment. Third, a single tumor biopsy often does not represent the whole tumor heterogeneities, which represent a significant challenge leading to disease relapse. On the other hand, CTCs collected from distinct treatment stages may reveal the evolving tumor characteristics and potentially capture the dynamic evolution of the tumor, which may provide important clues in devising more effective cancer therapies. While other liquid biopsies, such as circulation tumor DNA (ctDNA) and exosomes, may similarly serve the purpose in guiding therapeutic decisions during cancer detection and treatment (Pantel and Alix-Panabieres, 2019; Kilgour et al., 2020; Pessoa et al., 2020; Ignatiadis et al., 2021; Yu et al., 2021), CTCs appear to be unique for their functional role as the major metastases-seeding cells. In the next sections, we aim to provide a snapshot of the recent advances in CTC biological studies and how these new findings may be translated into clinical applications.

\section{BIOLOGICAL ANALYSES OF CIRCULATING TUMOR CELLS}

Although immunostaining and enumeration of CTCs remain effective in predicting disease progression and therapeutic response in a variety of cancer types (Cristofanilli et al., 2004; Sastre et al., 2008; Scher et al., 2009; Riethdorf et al., 2010; 


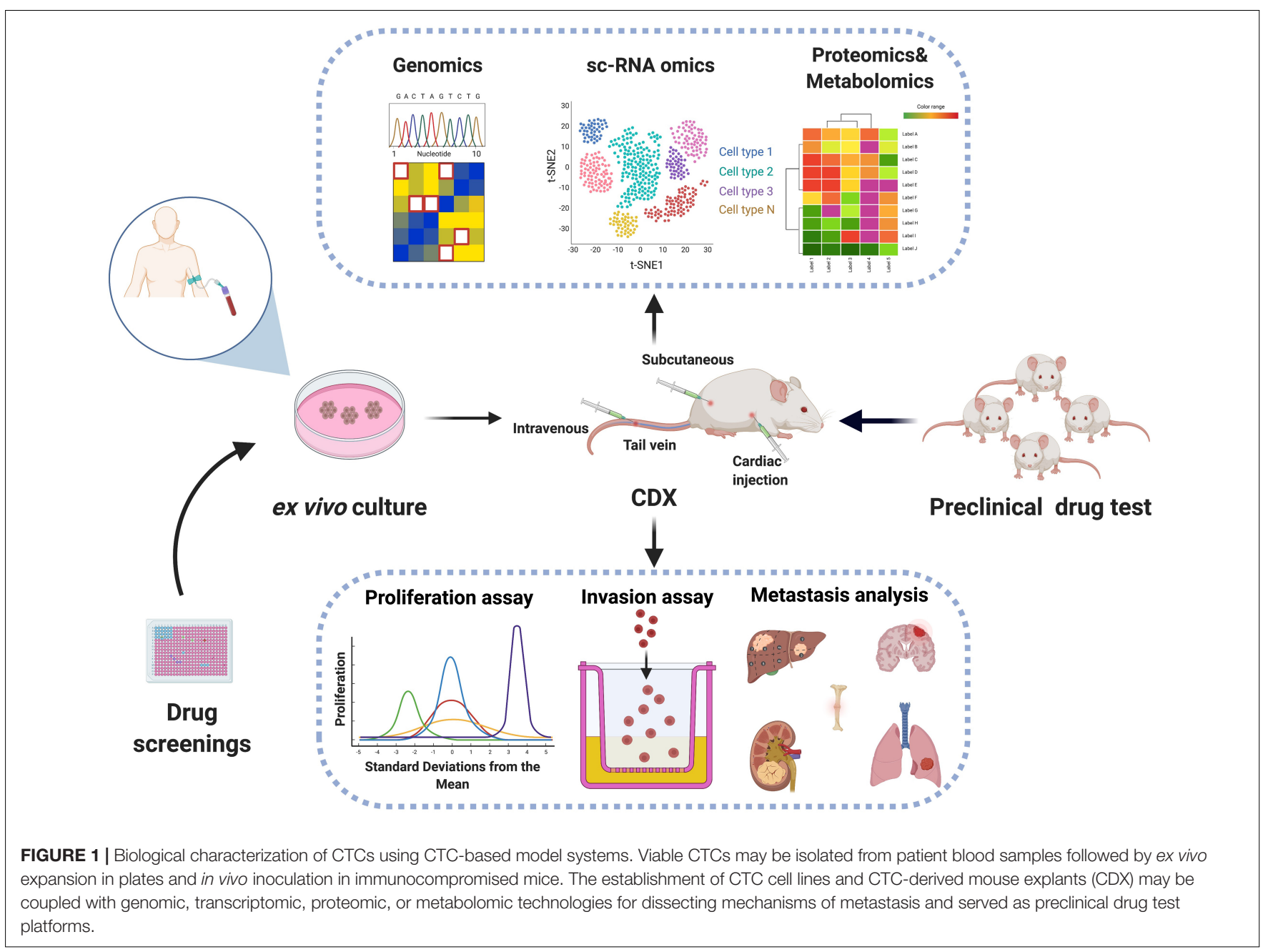

Cristofanilli et al., 2019), it is the recent technological revolutions on microfluidic engineering techniques and single-cell omic studies that begin to unravel the molecular "dark matter" of these "metastatic precursor" populations. Using high-resolution single-cell analyses, it is now possible to systematically profile the genome, transcriptome, and proteome of individually isolated CTCs or established CTC lines, which may provide remarkable insights into CTC-mediated metastatic process (Krebs et al., 2014; Micalizzi et al., 2017).

Genomic and transcriptomic studies using single-cell RNAseq have revealed considerable heterogeneities among individual CTCs and CTC subpopulations, in a manner that may be reflecting the multi-clonal origin of their matched tumors (Lohr et al., 2014; Miyamoto et al., 2015; Jordan et al., 2016; Lim et al., 2019; Riebensahm et al., 2019; Klotz et al., 2020; Hong et al., 2021). The Love lab performed a proof-of-concept study using whole-exome sequencing to characterize the genomic alterations of CTCs from metastatic prostate cancer patients and revealed both common and distinct mutational signatures by comparing CTCs with matched tumors, which may reflect the evolutionary dynamics during metastatic progression (Lohr et al., 2014). Miyamoto et al. carried out single-cell RNA-seq using 77 isolated prostate CTCs from 13 patients and uncovered striking heterogeneities within distinct CTC subpopulations, which contained acquired distinct androgen receptor (AR) gene mutations and differential splice variants expression. Molecular analyses of these cells further identified the correlation between the activation of non-canonical WNT signaling in CTC subclones and therapeutic resistance in patients treated with $\mathrm{AR}$ inhibitors (Miyamoto et al., 2015). Hodgkinson et al. (2014) established a panel of small cell lung cancer (SCLC)-derived explant (CDX) models for individualized drug testings, an approach that was also reported in ex vivo cultured breast CTC lines (Yu et al., 2014). Using breast CTC lines as well as fresh CTCs isolated from breast cancer patients that were initially diagnosed to be estrogen receptor-positive $(\mathrm{ER}+)$ and human epidermal growth factor 2 negative (HER2-), Jordan et al. (2016) identified heterogeneous CTC populations that co-existed as either HER2 ${ }^{+}$or HER2subclones. Remarkably, these two CTC subtypes were driven by distinct oncogenic signaling and can be inter-convertible upon differential treatment. Simultaneous targeting of both HER2 $^{+}$and HER2- CTC populations using inhibitors blocking both pathways resulted in the abolishment of CTC-mediated tumorigenesis in mouse xenografts (Jordan et al., 2016). Using 


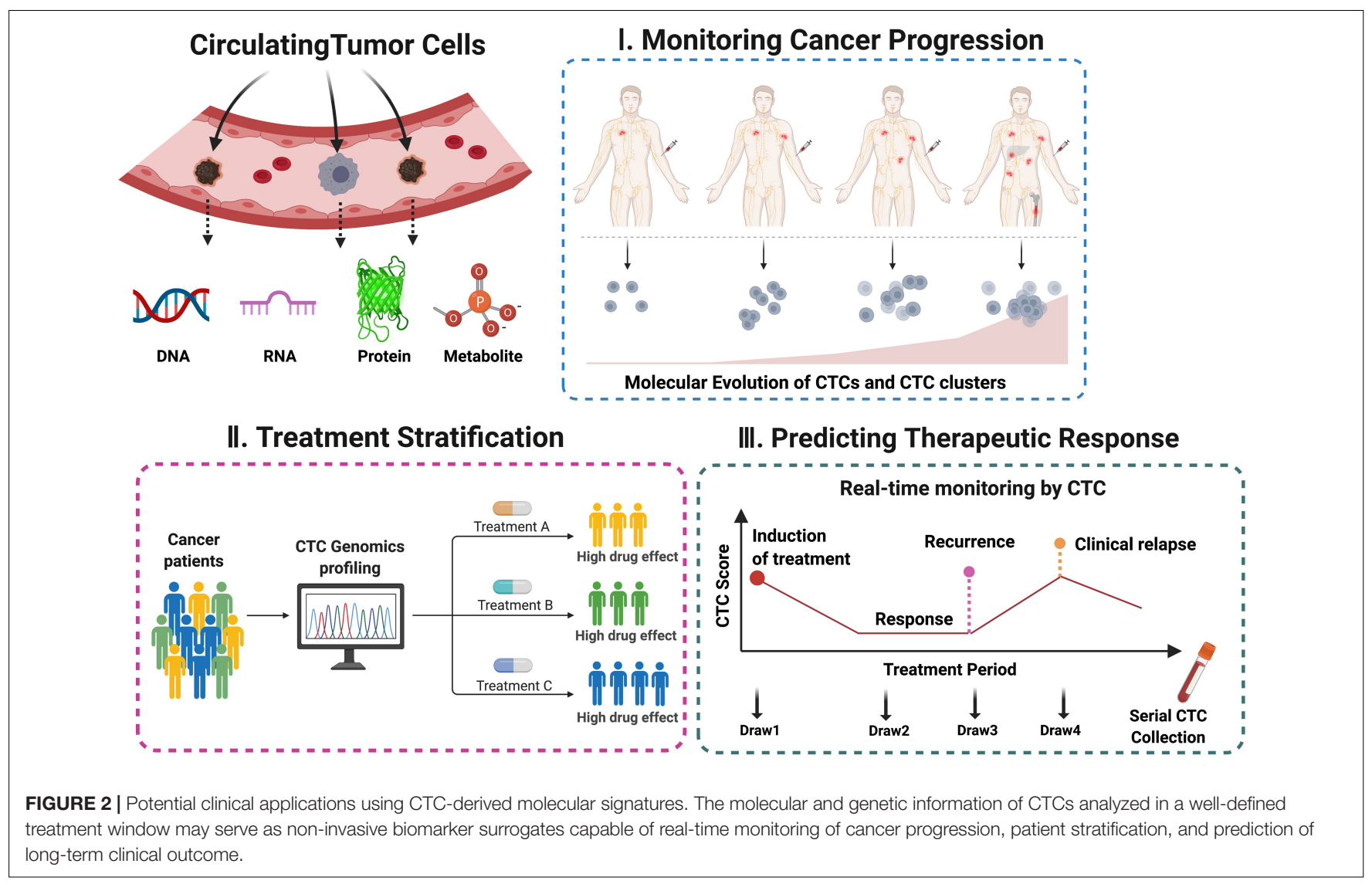

patient-derived CTC cell lines and mouse models, Klotz et al. (2020) identified Semaphorin 4D and Myc as site-specific drivers of brain metastases. Similarly, biological characterization of five established melanoma CTC lines and single-cell transcriptomic profiling of 76 primary CTCs isolated from 22 metastatic melanoma patients revealed the upregulation of SREBP-mediated lipogenesis in a subset of CTCs with higher proliferative potential, which was well-correlated with drug resistance and poor prognosis (Hong et al., 2021). These studies collectively demonstrated the power of CTC biological characterizations at single-cell levels in deciphering cancer heterogeneities and metastatic tropisms (Keller and Pantel, 2019).

It was initially discovered that some breast cancer patients had CTCs traveled along with white blood cells within the circulation called "CTC clusters" (Stott et al., 2010; Yu et al., 2013). Elegant studies coupling single-CTC profiling and mouse models have subsequently provided significant biological insights on these very rare but clinically important CTC subpopulations (Aceto et al., 2014; Gkountela et al., 2019; Szczerba et al., 2019; Donato et al., 2020). DNA methylome analyses of single CTC and CTC clusters uncovered hypomethylated regions that were selectively occupied by the key stemness transcription factors OCT4, NANOG, SOX2, and SIN3A, which may play critical roles in the metastatic seeding of CTC clusters (Gkountela et al., 2019). Further single-cell studies of CTC-associated white blood cells had pinpointed an unexpected role of neutrophils in promoting CTC cluster proliferation and metastatic colonization (Szczerba et al., 2019). Other heterotypic interactions of CTCs with other non-CTC cells such as cancer-associated stromal cells, fibroblasts, and certain immune cell types were also reported to have a significant impact on CTC survival or metastatic colonization (Duda et al., 2010; Labelle et al., 2011; Sprouse et al., 2019; Ortiz-Otero et al., 2020). Therefore, the establishment of CTC experimental systems including ex vivo-cultured CTC lines, CDX models, in conjunction with single-cell omic technologies may greatly accelerate the fundamental research on CTC biology, and open the door for the potential implementation of CTC-based clinical applications, such as CTC bio as companion diagnostics and novel therapies that selectively eliminate CTCs (Figure 1).

\section{CLINICAL TRANSLATIONS FROM CTC BIOLOGICAL DISCOVERIES}

The enumeration of CTCs has been clinically validated as an important prognosis factor in multiple cancers such as breast, prostate, and colorectal cancers in patients (Cristofanilli et al., 2004; Riethdorf et al., 2007; Sastre et al., 2008; Scher et al., 2009; Miller et al., 2010). However, its clinical utilities remain relatively limited given the vast degree of CTC heterogeneities, variabilities in CTC isolation technologies, and potential biases during CTC downstream molecular processing and manipulation steps that appear to vary from one study to another. Nevertheless, 
its clinical potential may be fully unleashed at a time when we are able to achieve a complete understanding of the biological properties of CTCs. With the abundance of molecular-level information derived from CTCs at the genomic, transcriptomic, proteomic, and metabolomic levels, CTC-based biomarker panels may be developed to precisely stratify patient and monitor disease monitoring following distinct therapeutic courses.

With the knowledge of cancer or CTC-specific transcriptomic studies, we and collaborators recently developed highly sensitive and specific CTC molecular assays using microfluidic enrichment of CTCs coupled with digital droplet PCR (ddPCR)-based profiling technologies (Kalinich et al., 2017; Hong et al., 2018; Kwan et al., 2018; Miyamoto et al., 2018). Individual transcripts that were abundant in CTCs but barely detectable in healthy donor white blood cells were quantified before and after specific therapies using serial blood collections. Such approaches revealed the predictive value of CTC signatures in several distinct cancer types under different treatment regimens. A 10gene CTC signature was applied to monitor the therapeutic efficacy of patients bearing hepatocellular carcinoma (HCC) and can be used to detect early HCC occurrence in the high-risk population (Kalinich et al., 2017). In a prospective cohort of metastatic melanoma patients receiving immune checkpoint inhibition therapies, 19-gene CTC RNA signatures were implemented to monitor early treatment response, which showed a strong predictive value of long-term clinical outcome in two independent clinical studies (Hong et al., 2018; AyaBonilla et al., 2020). Similarly, a panel of prostate CTC-specific RNA signatures was assessed for its ability to guide treatment selection in both localized and metastatic prostate cancer patients (Miyamoto et al., 2018). Such CTC quantification strategy was also demonstrated to be effective in inferring treatment response in both localized and metastatic breast cancer patients (Kwan et al., 2018).

In addition to RNA-level molecular profiling, quantification of CTC-specific DNA copy number alterations (CNA) was applied to stratify chemo-sensitive and resistant patients in small cell lung cancer (SCLC) (Carter et al., 2017). Direct monitoring of T790M mutation in both CTCs and ctDNAs were employed to potentially guide treatment selection on EGFR-mutant nonsmall cell lung cancer (NSCLC) patients (Sundaresan et al., 2016). Whole-genome sequencing of single CTCs isolated from NSCLC patients revealed mutational signatures that were shared with metastases that were found 10 months after CTC analyses. Remarkably, these signatures were not detectable in matched primary tumors, suggesting the potential utility of CTC genomic signatures in inferring metastatic propensity and disease relapse (Chemi et al., 2019). Besides these transcriptomic and genomic profiling methods, recent studies have also attempted to perform proteomic or metabolomic characterizations of individual CTCs using microfluidics or mass spectrometry-based technologies ( $\mathrm{Li}$ et al., 2015; Spitzer and Nolan, 2016; Sinkala et al., 2017; Abouleila et al., 2019; Liu et al., 2019). Although more independent clinical validations with a larger cohort of patient studies are needed, these reports provide the proof-of-principle evidence that CTC molecular signatures may be translated into clinical applications for the accurate stratification of patients, precise monitoring of cancer progression and prediction of therapeutic response throughout the entire treatment courses (Figure 2).

\section{CONCLUSION AND FUTURE PERSPECTIVES}

The recent advances in microfluidics enable the efficient isolation of viable CTCs that could be propagated by ex vivo culture and in vivo animal models (Lallo et al., 2017; Pei et al., 2020). Single-cell sequencing technologies have greatly accelerated the biological discoveries of CTCs. The establishment of CTC-based experimental systems together with single-cell omic profilings will undoubtedly pave the way to decipher the molecular dynamics of CTC-mediated metastatic evolution. Such comprehensive understanding of CTC biology is critically important for devising effective CTC biomarker panels for personalized cancer monitoring and diagnosis, while novel treatment strategies targeting CTCs may represent a new dimension of cancer therapies. Yet, several issues remain to be resolved before CTC liquid biopsies could be truly brought to clinical practice. More efficient and scalable CTC isolation platforms should be developed, so that viable CTCs could be routinely recovered in a variety of solid cancer types, but not just on the few reported cancer types thus far. The success rate of CTC culture and preclinical CDX models remains very low that warrants further improvement before they could be used for longitudinal studies in patients. As a result, the current collections of CTC cell lines or CDX models are still far too limited for conducting systematic drug studies. Further, the sensitivity and specificity of current CTC biomarker panels require substantial improvement. Novel ultra-high throughput quantification strategies that may enable simultaneous profiling of multi-marker panels are yet to be developed to provide comprehensive coverage of these highly heterogenous cancer cell subpopulations. Despite these challenges, the development of CTC-based liquid biopsies is rapidly moving forward, which may dramatically expand our knowledge of cancer metastases and ultimately change the clinical practice of cancer management.

\section{AUTHOR CONTRIBUTIONS}

JaL and XH conceptualized and wrote the manuscript. All authors contributed to the writing and revision of the manuscript.

\section{FUNDING}

This work was supported by the Guangdong Provincial Medical Research Funding A2021032 (granted to XH).

\section{ACKNOWLEDGMENTS}

We thank Ms. Xinran Liu for the great technical assistant and Southern University of Science and Technology (SUSTech) for providing start-up grant support to $\mathrm{XH}$. 


\section{REFERENCES}

Abouleila, Y., Onidani, K., Ali, A., Shoji, H., Kawai, T., Lim, C. T., et al. (2019). Live single cell mass spectrometry reveals cancer-specific metabolic profiles of circulating tumor cells. Cancer Sci. 110, 697-706. doi: 10.1111/cas.13915

Aceto, N., Bardia, A., Miyamoto, D. T., Donaldson, M. C., Wittner, B. S., Spencer, J. A., et al. (2014). Circulating tumor cell clusters are oligoclonal precursors of breast cancer metastasis. Cell 158, 1110-1122. doi: 10.1016/j.cell.2014.07.013

Alix-Panabieres, C. (2020). The future of liquid biopsy. Nature 579:S9.

Aya-Bonilla, C. A., Morici, M., Hong, X., McEvoy, A. C., Sullivan, R. J., Freeman, J., et al. (2020). Detection and prognostic role of heterogeneous populations of melanoma circulating tumour cells. Br. J. Cancer 122, 1059-1067. doi: 10.1038/s41416-020-0750-9

Banko, P., Lee, S. Y., Nagygyorgy, V., Zrinyi, M., Chae, C. H., Cho, D. H., et al. (2019). Technologies for circulating tumor cell separation from whole blood. J. Hematol. Oncol. 12:48.

Carter, L., Rothwell, D. G., Mesquita, B., Smowton, C., Leong, H. S., FernandezGutierrez, F., et al. (2017). Molecular analysis of circulating tumor cells identifies distinct copy-number profiles in patients with chemosensitive and chemorefractory small-cell lung cancer. Nat. Med. 23, 114-119. doi: 10.1038/ nm.4239

Chemi, F., Rothwell, D. G., McGranahan, N., Gulati, S., Abbosh, C., Pearce, S. P., et al. (2019). Pulmonary venous circulating tumor cell dissemination before tumor resection and disease relapse. Nat. Med. 25, 1534-1539. doi: 10.1038/ s41591-019-0593-1

Cristofanilli, M., Budd, G. T., Ellis, M. J., Stopeck, A., Matera, J., Miller, M. C., et al. (2004). Circulating tumor cells, disease progression, and survival in metastatic breast cancer. N. Engl. J. Med. 351, 781-791.

Cristofanilli, M., Pierga, J. Y., Reuben, J., Rademaker, A., Davis, A. A., Peeters, D. J., et al. (2019). The clinical use of circulating tumor cells (CTCs) enumeration for staging of metastatic breast cancer (MBC): international expert consensus paper. Crit. Rev. Oncol. Hematol. 134, 39-45.

Donato, C., Kunz, L., Castro-Giner, F., Paasinen-Sohns, A., Strittmatter, K., Szczerba, B. M., et al. (2020). Hypoxia Triggers the Intravasation of Clustered Circulating Tumor Cells. Cell Rep. 32:108105. doi: 10.1016/j.celrep.2020. 108105

Drapkin, B. J., George, J., Christensen, C. L., Mino-Kenudson, M., Dries, R., Sundaresan, T., et al. (2018). Genomic and Functional Fidelity of Small Cell Lung Cancer Patient-Derived Xenografts. Cancer Discov. 8, 600-615.

Duda, D. G., Duyverman, A. M., Kohno, M., Snuderl, M., Steller, E. J., Fukumura, D., et al. (2010). Malignant cells facilitate lung metastasis by bringing their own soil. Proc. Natl. Acad. Sci. U. S. A. 107, 21677-82. doi: 10.1073/pnas.1016234107

Ferreira, M. M., Ramani, V. C., and Jeffrey, S. S. (2016). Circulating tumor cell technologies. Mol. Oncol. 10, 374-394.

Gkountela, S., Castro-Giner, F., Szczerba, B. M., Vetter, M., Landin, J., Scherrer, R., et al. (2019). Circulating Tumor Cell Clustering Shapes DNA Methylation to Enable Metastasis Seeding. Cell 176, 98-112.e14.

Haber, D. A., and Velculescu, V. E. (2014). Blood-based analyses of cancer: circulating tumor cells and circulating tumor DNA. Cancer Discov. 4, 650-661. doi: 10.1158/2159-8290.cd-13-1014

Hanahan, D., and Weinberg, R. A. (2011). Hallmarks of cancer: the next generation. Cell 144, 646-674. doi: 10.1016/j.cell.2011.02.013

Hodgkinson, C. L., Morrow, C. J., Li, Y., Metcalf, R. L., Rothwell, D. G., Trapani, F., et al. (2014). Tumorigenicity and genetic profiling of circulating tumor cells in small-cell lung cancer. Nat. Med. 20, 897-903.

Hong, X., Roh, W., Sullivan, R. J., Wong, K. H. K., Wittner, B. S., Guo, H., et al. (2021). The Lipogenic Regulator SREBP2 Induces Transferrin in Circulating Melanoma Cells and Suppresses Ferroptosis. Cancer Discov. 11, 678-695. doi: 10.1158/2159-8290.cd-19-1500

Hong, X., Sullivan, R. J., Kalinich, M., Kwan, T. T., Giobbie-Hurder, A., Pan, S., et al. (2018). Molecular signatures of circulating melanoma cells for monitoring early response to immune checkpoint therapy. Proc. Natl. Acad. Sci. U. S. A. 115, 2467-2472. doi: 10.1073/pnas.1719264115

Hunter, K. W., Amin, R., Deasy, S., Ha, N. H., and Wakefield, L. (2018). Genetic insights into the morass of metastatic heterogeneity. Nat. Rev. Cancer 18, 211-223. doi: $10.1038 /$ nrc. 2017.126
Ignatiadis, M., Sledge, G. W., and Jeffrey, S. S. (2021). Liquid biopsy enters the clinic - implementation issues and future challenges. Nat. Rev. Clin. Oncol. 18, 297-312. doi: 10.1038/s41571-020-00457-x

Jackson, J. M., Witek, M. A., Kamande, J. W., and Soper, S. A. (2017). Materials and microfluidics: enabling the efficient isolation and analysis of circulating tumour cells. Chem. Soc. Rev. 46, 4245-4280. doi: 10.1039/c7cs00016b

Jordan, N. V., Bardia, A., Wittner, B. S., Benes, C., Ligorio, M., Zheng, Y., et al. (2016). HER2 expression identifies dynamic functional states within circulating breast cancer cells. Nature 537, 102-106. doi: 10.1038/nature19328

Kalinich, M., Bhan, I., Kwan, T. T., Miyamoto, D. T., Javaid, S., LiCausi, J. A., et al. (2017). An RNA-based signature enables high specificity detection of circulating tumor cells in hepatocellular carcinoma. Proc. Natl. Acad. Sci. U. S. A. 114, 1123-1128. doi: 10.1073/pnas.1617032114

Kallergi, G., Politaki, E., Alkahtani, S., Stournaras, C., and Georgoulias, V. (2016). Evaluation of Isolation Methods for Circulating Tumor Cells (CTCs). Cell Physiol. Biochem. 40, 411-419. doi: 10.1159/000452556

Karabacak, N. M., Spuhler, P. S., Fachin, F., Lim, E. J., Pai, V., Ozkumur, E., et al. (2014). Microfluidic, marker-free isolation of circulating tumor cells from blood samples. Nat. Protoc. 9, 694-710.

Keller, L., and Pantel, K. (2019). Unravelling tumour heterogeneity by singlecell profiling of circulating tumour cells. Nat. Rev. Cancer 19, 553-567. doi: 10.1038/s41568-019-0180-2

Kilgour, E., Rothwell, D. G., Brady, G., and Dive, C. (2020). Liquid Biopsy-Based Biomarkers of Treatment Response and Resistance. Cancer Cell 37, 485-495. doi: 10.1016/j.ccell.2020.03.012

Klotz, R., Thomas, A., Teng, T., Han, S. M., Iriondo, O., Li, L., et al. (2020). Circulating Tumor Cells Exhibit Metastatic Tropism and Reveal Brain Metastasis Drivers. Cancer Discov. 10, 86-103. doi: 10.1158/2159-8290.cd-190384

Krebs, M. G., Metcalf, R. L., Carter, L., Brady, G., Blackhall, F. H., and Dive, C. (2014). Molecular analysis of circulating tumour cells-biology and biomarkers. Nat. Rev. Clin. Oncol. 11, 129-144. doi: 10.1038/nrclinonc.2013.253

Kwan, T. T., Bardia, A., Spring, L. M., Giobbie-Hurder, A., Kalinich, M., Dubash, T., et al. (2018). A Digital RNA Signature of Circulating Tumor Cells Predicting Early Therapeutic Response in Localized and Metastatic Breast Cancer. Cancer Discov. 8, 1286-1299. doi: 10.1158/2159-8290.cd-18-0432

Labelle, M., Begum, S., and Hynes, R. O. (2011). Direct signaling between platelets and cancer cells induces an epithelial-mesenchymal-like transition and promotes metastasis. Cancer Cell 20, 576-590. doi: 10.1016/j.ccr.2011.09.009

Lallo, A., Schenk, M. W., Frese, K. K., Blackhall, F., and Dive, C. (2017). Circulating tumor cells and CDX models as a tool for preclinical drug development. Transl. Lung Cancer Res. 6, 397-408. doi: 10.21037/tlcr.2017.08.01

Lambert, A. W., Pattabiraman, D. R., and Weinberg, R. A. (2017). Emerging Biological Principles of Metastasis. Cell 168, 670-691. doi: 10.1016/j.cell.2016. 11.037

Li, S., Plouffe, B. D., Belov, A. M., Ray, S., Wang, X., Murthy, S. K., et al. (2015). An Integrated Platform for Isolation, Processing, and Mass Spectrometry-based Proteomic Profiling of Rare Cells in Whole Blood. Mol. Cell Proteomics 14, 1672-1683. doi: 10.1074/mcp.m114.045724

Li, W., Wang, H., Zhao, Z., Gao, H., Liu, C., Zhu, L., et al. (2019). Emerging Nanotechnologies for Liquid Biopsy: the Detection of Circulating Tumor Cells and Extracellular Vesicles. Adv. Mater. 31:e1805344.

Lim, S. B., Yeo, T., Lee, W. D., Bhagat, A. A. S., Tan, S. J., Tan, D. S. W., et al. (2019). Addressing cellular heterogeneity in tumor and circulation for refined prognostication. Proc. Natl. Acad. Sci. U. S. A. 116, 17957-17962. doi: 10.1073/ pnas.1907904116

Lin, Z., Luo, G., Du, W., Kong, T., Liu, C., and Liu, Z. (2020). Recent Advances in Microfluidic Platforms Applied in Cancer Metastasis: circulating Tumor Cells' (CTCs) Isolation and Tumor-On-A-Chip. Small 16:e1903899.

Liu, Y., Chen, X., Zhang, Y., and Liu, J. (2019). Advancing single-cell proteomics and metabolomics with microfluidic technologies. Analyst 144, 846-858. doi: 10.1039/c8an01503a

Lohr, J. G., Adalsteinsson, V. A., Cibulskis, K., Choudhury, A. D., Rosenberg, M., Cruz-Gordillo, P., et al. (2014). Whole-exome sequencing of circulating tumor cells provides a window into metastatic prostate cancer. Nat. Biotechnol. 32, 479-484. 
McGranahan, N., and Swanton, C. (2017). Clonal Heterogeneity and Tumor Evolution: past, Present, and the Future. Cell 168, 613-628. doi: 10.1016/j.cell. 2017.01.018

Micalizzi, D. S., Haber, D. A., and Maheswaran, S. (2017). Cancer metastasis through the prism of epithelial-to-mesenchymal transition in circulating tumor cells. Mol. Oncol. 11, 770-780. doi: 10.1002/1878-0261.12081

Miller, M. C., Doyle, G. V., and Terstappen, L. W. (2010). Significance of Circulating Tumor Cells Detected by the CellSearch System in Patients with Metastatic Breast Colorectal and Prostate Cancer. J. Oncol. 2010:617421.

Miyamoto, D. T., Lee, R. J., Kalinich, M., LiCausi, J. A., Zheng, Y., Chen, T., et al. (2018). An RNA-Based Digital Circulating Tumor Cell Signature Is Predictive of Drug Response and Early Dissemination in Prostate Cancer. Cancer Discov. 8, 288-303. doi: 10.1158/2159-8290.cd-16-1406

Miyamoto, D. T., Zheng, Y., Wittner, B. S., Lee, R. J., Zhu, H., Broderick, K. T., et al. (2015). RNA-Seq of single prostate CTCs implicates noncanonical Wnt signaling in antiandrogen resistance. Science 349, 1351-1356. doi: 10.1126/ science.aab0917

Nguyen, D. X., Bos, P. D., and Massague, J. (2009). Metastasis: from dissemination to organ-specific colonization. Nat. Rev. Cancer 9, 274-284. doi: 10.1038/ $\operatorname{nrc} 2622$

Ortiz-Otero, N., Clinch, A. B., Hope, J., Wang, W., Reinhart-King, C. A., and King, M. R. (2020). Cancer associated fibroblasts confer shear resistance to circulating tumor cells during prostate cancer metastatic progression. Oncotarget. 11, 1037-1050. doi: 10.18632/oncotarget.27510

Ozkumur, E., Shah, A. M., Ciciliano, J. C., Emmink, B. L., Miyamoto, D. T., Brachtel, E., et al. (2013). Inertial focusing for tumor antigen-dependent and independent sorting of rare circulating tumor cells. Sci. Transl. Med. 5:179ra47.

Pantel, K., and Alix-Panabieres, C. (2019). Liquid biopsy and minimal residual disease - latest advances and implications for cure. Nat. Rev. Clin. Oncol. 16, 409-424. doi: 10.1038/s41571-019-0187-3

Pantel, K., Alix-Panabieres, C., and Riethdorf, S. (2009). Cancer micrometastases. Nat. Rev. Clin. Oncol. 6, 339-351.

Pantel, K., and Speicher, M. R. (2016). The biology of circulating tumor cells. Oncogene 35, 1216-1224.

Papadaki, M. A., Stoupis, G., Theodoropoulos, P. A., Mavroudis, D., Georgoulias, V., and Agelaki, S. (2019). Circulating Tumor Cells with Stemness and Epithelial-to-Mesenchymal Transition Features Are Chemoresistant and Predictive of Poor Outcome in Metastatic Breast Cancer. Mol. Cancer Ther. 18, 437-447. doi: 10.1158/1535-7163.mct-18-0584

Pei, H., Li, L., Han, Z., Wang, Y., and Tang, B. (2020). Recent advances in microfluidic technologies for circulating tumor cells: enrichment, single-cell analysis, and liquid biopsy for clinical applications. Lab Chip 20, 3854-3875. doi: $10.1039 / \mathrm{d} 01 \mathrm{lc} 00577 \mathrm{k}$

Pessoa, L. S., Heringer, M., and Ferrer, V. P. (2020). ctDNA as a cancer biomarker: a broad overview. Crit. Rev. Oncol. Hematol. 155:103109. doi: 10.1016/j. critrevonc.2020.103109

Riebensahm, C., Joosse, S. A., Mohme, M., Hanssen, A., Matschke, J., Goy, Y., et al. (2019). Clonality of circulating tumor cells in breast cancer brain metastasis patients. Breast Cancer Res. 21:101.

Riethdorf, S., Fritsche, H., Muller, V., Rau, T., Schindlbeck, C., Rack, B., et al. (2007). Detection of circulating tumor cells in peripheral blood of patients with metastatic breast cancer: a validation study of the CellSearch system. Clin. Cancer Res. 13, 920-928. doi: 10.1158/1078-0432.ccr-06-1695

Riethdorf, S., Muller, V., Zhang, L., Rau, T., Loibl, S., Komor, M., et al. (2010). Detection and HER2 expression of circulating tumor cells: prospective monitoring in breast cancer patients treated in the neoadjuvant GeparQuattro trial. Clin. Cancer Res. 16, 2634-2645. doi: 10.1158/1078-0432.ccr-09-2042

Sastre, J., Maestro, M. L., Puente, J., Veganzones, S., Alfonso, R., Rafael, S., et al. (2008). Circulating tumor cells in colorectal cancer: correlation with clinical and pathological variables. Ann. Oncol. 19, 935-938. doi: 10.1093/annonc/mdm583
Scher, H. I., Jia, X., de Bono, J. S., Fleisher, M., Pienta, K. J., Raghavan, D., et al. (2009). Circulating tumour cells as prognostic markers in progressive, castration-resistant prostate cancer: a reanalysis of IMMC38 trial data. Lancet Oncol. 10, 233-239. doi: 10.1016/s1470-2045(08)70340-1

Sinkala, E., Sollier-Christen, E., Renier, C., Rosas-Canyelles, E., Che, J., Heirich, K., et al. (2017). Profiling protein expression in circulating tumour cells using microfluidic western blotting. Nat. Commun. 8:14622.

Spitzer, M. H., and Nolan, G. P. (2016). Mass Cytometry: single Cells, Many Features. Cell 165, 780-791. doi: 10.1016/j.cell.2016.04.019

Sprouse, M. L., Welte, T., Boral, D., Liu, H. N., Yin, W., Vishnoi, M., et al. (2019). PMN-MDSCs Enhance CTC Metastatic Properties through Reciprocal Interactions via ROS/Notch/Nodal Signaling. Int. J. Mol. Sci. 20:1916. doi: 10.3390/ijms20081916

Stott, S. L., Hsu, C. H., Tsukrov, D. I., Yu, M., Miyamoto, D. T., Waltman, B. A., et al. (2010). Isolation of circulating tumor cells using a microvortexgenerating herringbone-chip. Proc. Natl. Acad. Sci. U. S. A. 107, 18392-18397. doi: $10.1073 /$ pnas. 1012539107

Sundaresan, T. K., Sequist, L. V., Heymach, J. V., Riely, G. J., Janne, P. A., Koch, W. H., et al. (2016). Detection of T790M, the Acquired Resistance EGFR Mutation, by Tumor Biopsy versus Noninvasive Blood-Based Analyses. Clin. Cancer Res. 22, 1103-1110. doi: 10.1158/1078-0432.ccr-151031

Sung, H., Ferlay, J., Siegel, R. L., Laversanne, M., Soerjomataram, I., Jemal, A., et al. (2021). Global Cancer Statistics 2020: GLOBOCAN Estimates of Incidence and Mortality Worldwide for 36 Cancers in 185 Countries. CA Cancer J. Clin. 71, 209-249. doi: 10.3322/caac. 21660

Szczerba, B. M., Castro-Giner, F., Vetter, M., Krol, I., Gkountela, S., Landin, J., et al. (2019). Neutrophils escort circulating tumour cells to enable cell cycle progression. Nature 566, 553-557. doi: 10.1038/s41586-019-0915-y

Yu, M., Bardia, A., Aceto, N., Bersani, F., Madden, M. W., Donaldson, M. C., et al. (2014). Cancer therapy. Ex vivo culture of circulating breast tumor cells for individualized testing of drug susceptibility. Science 345, 216-220. doi: 10.1126/science. 1253533

Yu, M., Bardia, A., Wittner, B. S., Stott, S. L., Smas, M. E., Ting, D. T., et al. (2013). Circulating breast tumor cells exhibit dynamic changes in epithelial and mesenchymal composition. Science 339, 580-584. doi: 10.1126/science. 1228522

Yu, M., Stott, S., Toner, M., Maheswaran, S., and Haber, D. A. (2011). Circulating tumor cells: approaches to isolation and characterization. J. Cell Biol. 192, 373-382. doi: 10.1083/jcb.201010021

Yu, W., Hurley, J., Roberts, D., Chakrabortty, S. K., Enderle, D., Noerholm, M., et al. (2021). Exosome-based liquid biopsies in cancer: opportunities and challenges. Ann. Oncol. 32, 466-477. doi: 10.1016/j.annonc.2021.01.074

Conflict of Interest: The authors declare that the research was conducted in the absence of any commercial or financial relationships that could be construed as a potential conflict of interest.

Publisher's Note: All claims expressed in this article are solely those of the authors and do not necessarily represent those of their affiliated organizations, or those of the publisher, the editors and the reviewers. Any product that may be evaluated in this article, or claim that may be made by its manufacturer, is not guaranteed or endorsed by the publisher.

Copyright (c) 2021 Liu, Lian, Chen, Zhao, Du, Xu, Hu, Rao and Hong. This is an open-access article distributed under the terms of the Creative Commons Attribution License (CC BY). The use, distribution or reproduction in other forums is permitted, provided the original author(s) and the copyright owner(s) are credited and that the original publication in this journal is cited, in accordance with accepted academic practice. No use, distribution or reproduction is permitted which does not comply with these terms. 